\title{
Engine Dismounting and Operation Test Bench Design Based on Practice Ability Training
}

\section{YAN Wen-bing}

School of Automobile and transportation, Tianjin University of Technology and Education, Tianjin, China

ywenbing@126.com

Keywords: engine; test bench; dismounting and operation; practice ability.

\begin{abstract}
Based on corolla 1ZR electronic-controlled engine, design and research were made on the engine test bench with dismounting and operation function. The equipment can be used in operation teaching area of the auto repair industry or automotive related majors in vocational school. The bench includes the panel circuit, control circuit, fault points and the platform etc. It owns functions of the disassembling, inspection and fault diagnosis which are convenient for teachers integrated field teaching and can improve students' operation ability effectively.
\end{abstract}

\section{基于实践能力培养的发动机拆装运行实验台设计与实现}

\author{
阎文兵 \\ 天津职业技术师范大学汽车与交通学院, 天津, 中国 \\ ywenbing@126.com
}

关键词: 发动机; 实验台; 拆装运行; 实践能力

中文摘要. 本文以卡罗拉1ZR电控发动机为研究对象, 针对汽车维修行业从业人员或汽车相关 专业学生, 设计与研究了可适用于汽车实训教学的发动机拆装运行实验台。所设计的实验台 由面板电路、控制电路、故障点和台架等部分组成，具有发动机拆装、检测和故障诊断的功 能, 方便教师开展一体化现场教学, 有效提高学生实践能力。

\section{1. 引言}

随着汽车技术的日趋发展，汽车检测与维修行业对从业人员的基本素质和职业技能也提 出了更高的要求。汽车发动机拆装运行实验台就是针对当前各职业院校或培训基地培养培训 汽车技术人员开发的。

本设计采用卡罗拉1ZR发动机，该发动机具有电控燃油喷射系统、单缸独立点火系统、 电控急速系统以及可变气门正时系统等先进的发动机管理系统, 使发动机的动力性、经济性 和排放性达到最优控制。

所开发的实验台架是以教学为主, 运用该教学设备教师可以实现理实一体化现场教学, 学员能够把专业理论知识和实习实践相结合, 不仅符合职业学校汽车运用专业的人才培养目 标和教学特点, 而且很好的锻炼了学生的实践能力, 进一步提高了学生的专业技术能力和职 业综合素质，进一步达到企业职业岗位能力要求。 


\section{2. 实验台功能}

发动机拆装运行实验台具备拆装、故障设置、发动机信号采集的综合性能实验台。在实 训教学过程中, 教师可通过实验台对丰田花冠发动机的结构组成及工作原理进行讲解, 同时 可进行故障设置, 使学生在了解发动机的具体结构之后, 进行拆解发动机、组装发动机, 可 以在实验台上通过故障开关设置故障发动机的故障, 出现故障现象并可利用电脑故障诊断仪 和万用表采集发动机工作过程中的性能参数, 实现发动机综合故障分析、诊断与排除等功能。

\section{3. 实验台总体结构设计}

\section{1 设计思路}

(1) 确定发动机型号: 选定以丰田卡罗拉 $1 Z R$ 为设计对象。

（2）确定实验台的整体结构：面板电路、控制电路、故障点和台架。

(3) 实验台面板的设计：包括实验台名称、发动机电控系统电路图及检测点、诊断座。

(4) 控制面板的设计：控制电路、故障点连线等。

(5) 故障点和检测点: 根据发动机传感器与ECU之间的连线位置确定。

（6）实验台架的设计：根据发动机总体尺寸确定支撑台架的大小，根据支撑台架确定面 板的宽, 隐蔽式故障点设置在面板侧面, 并有罩盖。

\section{2 实验台组成}

根据实验台功能，汽车发动机拆装运行实验台架由发动机拆装台架和发动机检测台架两 大部分组成。发动机拆装台架是由发动机及支撑台架组成, 发动机检测台架由控制面板、故 障设置操作台、数据流检测端口、传感器显示屏幕、汽车仪表盘、ECU、点火开关、蓄电池、 油箱等组成。

发动机检测台架和发动机之间的连接是通过航空接头连接的。航空插头是一对配合件, 分别为公插头和母插头。ECU安装在检测台架箱体内, 发动机所有传感器和执行器的线束根 据原厂电路图进行编号, 并逐一焊接到航空接头公插头上。按照插头的对应关系, 将和ECU 相连的线束与航空接头母插头也逐一焊接在一起。

\section{3 实验台总体结构}

以方便实训教学，同时达到安全可靠、操作方便、结构稳定、造型美观的要求。实验台 框架如图1所示：采用45\#方刚（长宽为3.5CM）焊接制作成型, 表面经过喷塑处理, 能够防 腐、防锈, 台架底部安装有脚轮以方便移动。实验台总体长度为 $1800 \mathrm{~mm}$ 、高度为 $1600 \mathrm{~mm}$ 。

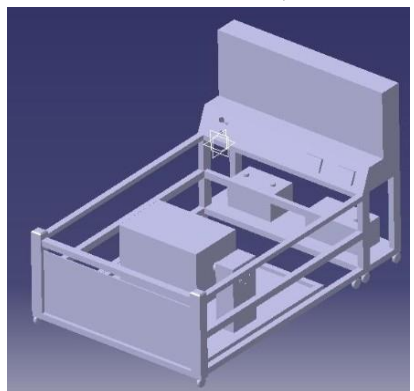

图 1 实验台结构

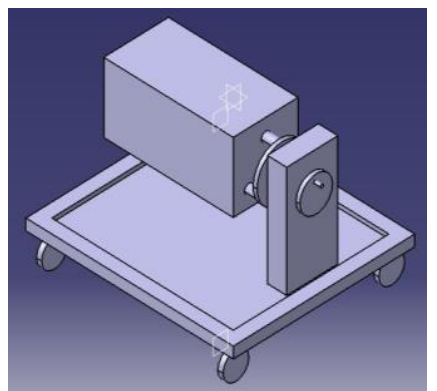

图 2 发动机拆装台架

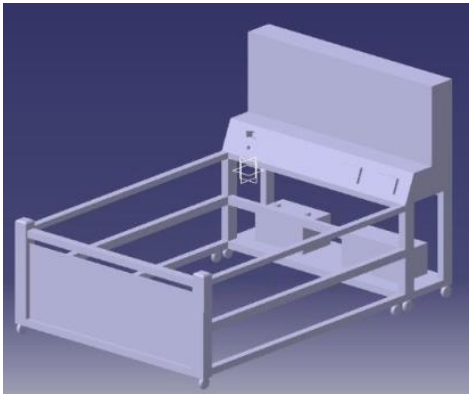

图 3 发动机检测台架

\section{4 发动机拆装台架}

发动机拆装台架具有独立的三角式脚轮, 可以独立运动, 如图2所示。该台架位于发动机 检测台架内, 学生进行拆装训练时, 将台架上边的锁扣开启, 发动机拆装台架可推至独立的 空间内进行操作。 


\section{5 发动机检测台架}

发动机检测组部分由一组元器件面板、故障设置操作台、数据流检测端口、传感器显示 屏幕、汽车仪表盘、ECU、点火开关、蓄电池、油箱等组成。学生可以将已经装配完整的1ZR 发动机拆装组与检测组部分连接, 继而对发动机实施故障检查。如图3所示。

发动机检测台架和发动机之间的连接是通过航空接头连接的。航空插头是一对配合件, 分别为公插头和母插头。ECU安装在检测台架箱体内, 发动机所有传感器和执行器的线束根 据原厂电路图进行编号, 并逐一焊接到航空接头公插头上。按照插头的对应关系, 将和ECU 相连的线束与航空接头母插头也逐一焊接在一起。

\section{4. 发动机检测台面板和控制台的设计}

\section{1 发动机检测台面板}

发动机检测台面板部分是进行实训教学、电路分析、故障诊断与排除的平台, 在面板上 还需要安装检测点、开关仪表等与后部控制箱内的线束连接, 其作用是直观地反映发动机在 工作过程中各传感器和执行器的工作情况, 同时为采用专用的仪器仪表检测发动机工作过程 中相关的性能参数设置相应的检测端口。发动机检测台面板是由实验台标题名称、部分发动 机传感器数据动态显示屏和花冠发动机控制电路图三部分组成。面板材料采用PCV双色板材, 实验台面板上的电路图采用 0.3 毫米和 1 毫米两种刻刀进行雕刻。

实验台面板上的电路图是根据丰田花冠汽车维修手册原件, 将各个执行器、传感器的原 理图经过分析组合起来, 要求各个电器原件均在电路图上有所显示, 并且电路完整。绘制出 的电路图必须清晰明了, 尽量避免出现电线交叉、跨线的情况, 以便后期的保养和维修。

\section{2 发动机检测台控制台}

控制部分是实验台的核心，包含发动机控制电脑ECU、点火开关，故障自诊断接口，故 障设置系统，显示系统和数据检测系统等。其作用是实现实验台的信号仿真、故障设置以及 数据显示和检测等功能。

发动机传感器上的线束经过故障开关并对照ECU端子进行端子匹配, 制作成故障点通过 线束焊接在发动机检测台架侧面的隐蔽故障开关板位置, 将仪表检测点等装入面板上，各部 分线路连接完整, 最后将稳压电源接入到仪表中, 本实验台共设置了 24 个故障点, 全部由手 动故障开关控制。

\section{3 直流数字电压面板表}

数字电压表又称数字面板表头, 它是采用数字化测量技术, 把连续的模拟量（直流输入 电压）转换成不连续、离散的数字形式并加以显示的仪表。显示面板上采用的 XL5135V-1 直 流数字电压面板表如图 4 所示, 由点火开关控制。当点火开关处于 $0 \mathrm{~N}$ 时, 通过控制显示表总 开关通断来控制数字电压表是否有数字显示。数字仪表的工作电压为直流 $5 \mathrm{~V}$ 。为了能够使其 正常工作，必须为其提供合适的工作电压。汽车上采用的是 $12 \mathrm{~V}$ 直流电，因此实验装置采用 78 系列集成稳压块 (三端集成稳压器) 将 $12 \mathrm{~V}$ 直流电变为 $5 \mathrm{~V}$ 直流电为仪表提供工作电源。

7805 是最常用到的稳压芯片, 它使用方便, 用很简单的电路即可以输入一个直流稳压电 源, 它的输出电压恰好为 $5 \mathrm{~V}$ 。 


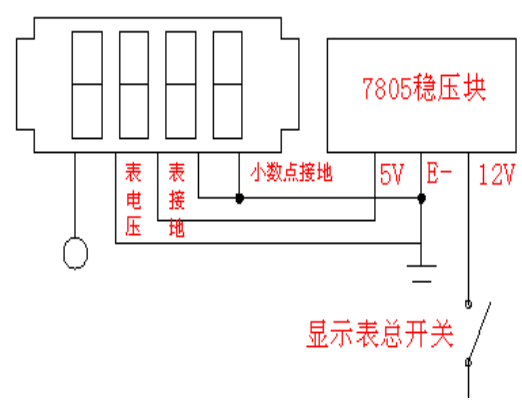

图 4 直流数字电压面板表

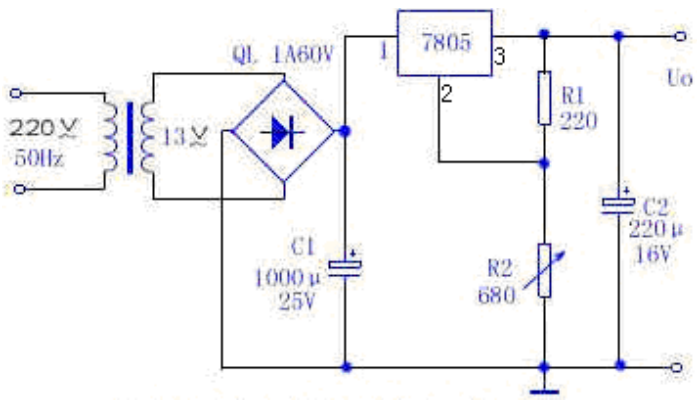

图 5 稳压器输出电压可调电路

图5中R1用 $220 \Omega$ 、 R2 用 $680 \Omega$ 的这个是用来调节稳压器的输出电压。输出电压公式 $U_{0} \approx$ $\mathrm{Uxx}(1+\mathrm{R} 2 / \mathrm{R} 1)$, 此稳压电路可在 $5 \sim 12 \mathrm{~V}$ 稳压范围内实现输出电压连续可调节。三端集成稳压 集成电路 7805 最大输入电压为 $35 \mathrm{~V}$, 输入与输出差需保持 $2 \mathrm{~V}$ 以上, 这样该电路中因为稳压器的 直流输入电压是正 $14 \mathrm{~V}$, 故该稳压电路的最大输出电压为正 $12 \mathrm{~V}$ 。此电路的精度一般可达到 0.04 以上，用7805就可满足一般需求了。

\section{5. 结束语}

本实验装置可以方便、直观地了解汽车发动机机械及电子控制系统的结构、组成和工作 原理, 可以过隐蔽故障点设置汽车发动机故障, 可以通过专用的仪器仪表检测发动机工作过 程中相关的性能参数, 进行故障诊断、检测与排除。为发动机构造与发动机管理系统的教、 学与实践提供了较好的支持。

\section{致谢}

本文为天津职业技术师范大汽车实验实训中心天津市实验示范建设中心阶段性成果之

\section{References}

[1] YU Chun-peng, LI Han-wu, BAO Yu, Design of an experimental platform for Toyota electronically-controled engine, Applied Science and Technology, vol.35,No.2, pp. 45-48, 2008.

[2] MA Jie, Design and application of automobile electronic control engine test bed, China Educational Technique \& Equipment, vol.23, pp.127-130, 2011.

[3] MEN Guo-qiang, ZHANG Yue-ying, Design of electronic control engine test bench, Journal of Zhengzhou Railway Vocational College, vol.17,No.3, pp.37-38, 200. 